\title{
Nature as a Model for Mimicking and Inspiration of New Technologies
}

\author{
Yoseph Bar-Cohen
}

Jet Propulsion Laboratory (JPL)/California Institute of Technology (Caltech) 4800 Oak Grove Drive, Pasadena, CA 91109-8099

\begin{abstract}
Over 3.8 billion years, through evolution nature came up with many effective continually improving solutions to its challenges. Humans have always been inspired by nature capabilities in problems solving and innovation. These efforts have been intensified in recent years where systematic studies are being made towards better understanding and applying more sophisticated capabilities in this field that is increasingly being titled biomimetics. The ultimate challenge to this field is the development of humanlike robots that talk, interpret speech, walk, as well as make eye-contact and facial expressions with some capabilities that are exceeding the original model from nature. This includes flight where there is no creature that is as large, can fly as high, carry so heavy weight, fly so fast, and able to operate in extreme conditions as the aircraft and other aerospace systems. However, there are many capabilities of biological systems that are not feasible to mimic using the available technology. In this paper, the state-of-the-art of some of the developed biomimetic capabilities, potentials and challenges will be reviewed.
\end{abstract}

Key words: Biologically inspired technologies, biomimetics, robotics, actuators, sensors, humanlike robots

\section{Introduction}

Nature is effectively a giant laboratory where trial and error experiments are taking place though evolution and the results are implemented, self-maintained and continually evolving to address the posed challenges. The experiments involve all the fields of science and engineering including physics, chemistry, mechanical engineering, materials science, and many others. They cover all the scale levels ranging from nano and micro (e.g., bacteria and viruses) to macro and mega (including our life scale and beyond). As opposed to the mega-size sea creatures such as the whales, independent of the cause, the giant creatures like the dinosaurs seem to be unsustainable form of life and they were extinct. Nature has always served as a model for humans' innovation and problems solving and the efforts have been intensified in recentyears. The field of science and engineering that seeks to understand nature as a model for copying, adapting and inspiring concepts and designs is now mostly called Biomimetics. As a model for inspiration, it is important to remember that Nature's solutions are the result of the survival of the fittest and these solutions are not necessarily optimal for the required function. Effectively, all organisms need to do is to survive long enough to reproduce.
The evolved and accumulated living system information is coded into the species' genes and passed from generation to generation through self-replication.

Many of Nature's materials and processes are far superior to man-made ones where the sea shells and the spider web are examples of such materials and structures. Benefiting from the up-to-date advances in science and technology we are significantlymoreableofstudyingthecapabilitiesandfunctions of Nature's inventions. The body of biological systems acts as a laboratory that processes chemicals from the surrounding to produce materials, energy, multifunctional structures, and waste [1-2]. Systematic studies of Nature are now extensively being made towards applying more sophisticated capabilities [3-6]. Scientists are seeking rules, concepts, mechanisms and principles of biology to inspire new engineering possibilities including manufacturing, mechanisms, materials, processes, and algorithms. The resulting benefits include improved structures, actuators, sensors, interfaces, control, software, drugs, defense, intelligence and many others.

Generally, even though humans are familiar with many of Nature's inventions they have not always adapted them to their needs. This suggests that we should be more proactive about studying and implementing Nature inventions. For

(c) E-mail : yosi@jpl.nasa.gov, web: http://ndeaa.jpl.nasa.gov 
example, the use of camouflage as an effective defense was very well known to humans from the many creatures that lived near their habitats. Predators conceal themselves to be able to reach as close to their prey with minimum probability of being seen until the desirable moment of striking. Further, preys reduce their visibility as much as possible to minimize the danger of being detected by potential predators. As an illustration of this capability, the lizard shown Figure 1 has colors and patterns that match the ground and trees on which it lives making its visibility very difficult unless it moves.

Plants also use camouflage to address their requirements. An example is the critical need of plants to protect their fruits before they are ripe and this is why unripe fruits are mostly green matching the color of the leaves. Once the fruit is ripe, it changes its color to make it as visible as possible (red, pink, yellow, or others). The photo in Figure 2 shows the lemon fruit as an example where among an assortment of unripe ones that are green the ripe one is yellow and easy to see. It is also interesting to note that when deciduous plants blossom, their flowers are colored and sized as visible as possible (beautiful colors, minimally obstructed, etc.). For many of them, once the flower is pollinated and fertilized the plant grows new leaves that cover the developing fruits and conceal them till they are ripe. Plants consist of many other "inventions" and the most famous one is the adherence of seeds to animals' fur, which led to the invention of the Velcro and the numerous applications including clothing and electric-wires strapping. Plants capability to distribute water evenly throughout their structure including at great heights as in giant trees offers important model for mimicking.

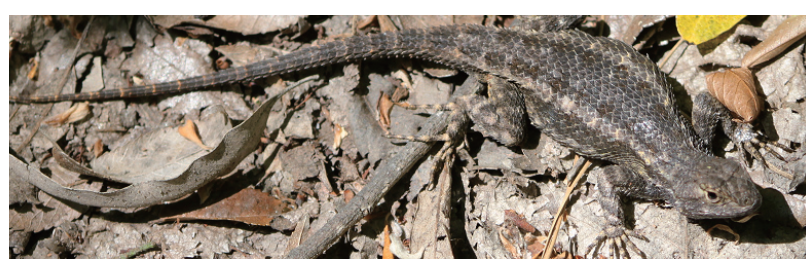

Fig. 1. The lizard has colors and pattern that match its surrounding to make it barely noticeable unless it moves.

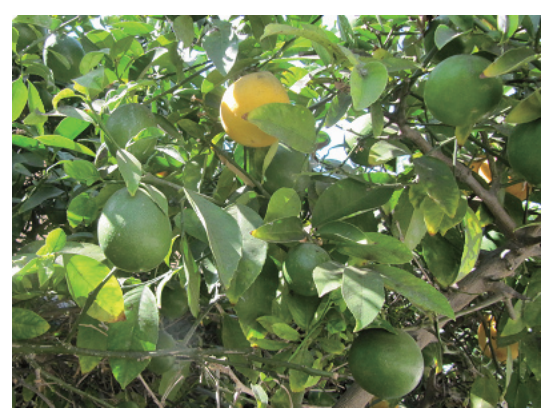

Fig. 2. The unripe lemon is camouflaged by staying green till it is ripe and turns yellow.
Moreover, the roots of plants are able to lift heavy structures as well as break rocks and mimicking these capabilities plants is quite a challenge [5-6].

One may wonder if the tools and capability that were used by humans over hundreds or thousand years ago were totally the result of humans' innovation or were mimicked from natures. Obviously, biological creatures evolved over many millions of years before humans reached the level of intelligence that was sufficient to start producing tools. Their produced tools resulted from the need to minimize the dependence on luck in finding food and resources. Observing nature most likely inspired many ideas and as humans' capability improved it became increasingly easier to mimic more sophisticated nature inventions. However, one may wonder which of the inventions and tools that resemble biological models and widely used by humans were the result of mimicking and which ones have just a coincident similarity. It is hard to believe that all humanmade solutions were purely independent inventions ignoring what is commonly seen in their neighborhood. For example, developing alternative to human breast for feeding the offsprings was critical for their babies' survival particularly in the cases of unavailability of the mother. This life-critical need has most likely inspired the bottle with soft nipple to make the baby receptive to this alternative feeding form. Following a similar logic, the spider web (Figure 3) should have had a contribution to human's making such things as wires, ropes, nets, sieves, screens and woven fabrics. One cannot ignore the similarity of the spider web to the fishing net, the screen in screen-doors, the kitchen strainer, or even our clothing.

\section{Biologically inspired technologies and mechanisms}

There are many examples of biologically inspired technologies that were developed in recent years including the following.
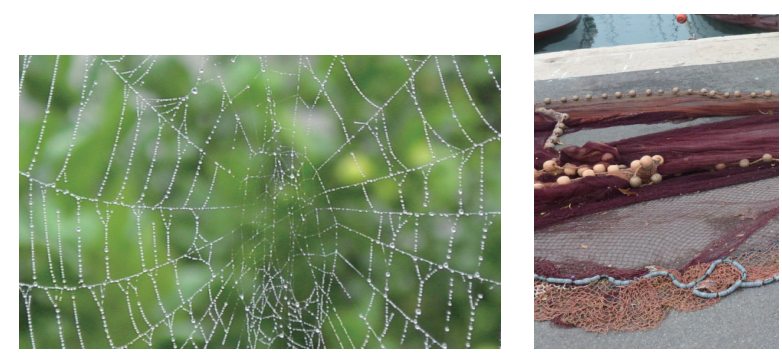

Fig. 3. The spider is an amazing "engineer" creating a large flat structure for trapping insects and it may have inspired the development of the fishing nets. 


\subsection{Artificial Intelligence (Al) and smart system con- trols}

The brain is an incredible controller of biological systems and the mimicking of its operation is increasingly being sought. The early developers of automation used software to control their developed systems and the software included predetermined options of actions and reactions. Increasingly, systems are being developed to operate "smarter" using artificial intelligence algorithms such as knowledge capture, representation and reasoning, planning, reasoning under uncertainty, vision, face and feature tracking, language processing, mapping and navigation, natural language processing, and machine learning [7-9]. These algorithms are used to perform such tasks as perception, reasoning, learning, and parallel processing $[10,11]$. For this purpose, AI models are used that are inspired by the computational capability of the brain and are explained in terms of higherlevel psychological constructs such as plans and goals.

\subsection{Artificial and biomimetic materials and struc- tures}

The body of organisms and creatures processes chemicals (food and drinks) and turn them into energy, construction materials, multifunctional structures, and waste [1]. The use of natural materials for human applications can be traced back many thousands of years and it includes the silk, bones, leather, fur, ivory and many others [12]. As a result of the recognition of these materials advantages there has been an ever-increasing need for supply and it led to the development of artificial versions. Besides their immediate use for thermal protection, food, cleaning, beauty, construction, etc., natural materials have other important capabilities many of which we don't know how to mimic. It is interesting to note that natural materials are normally produced in ambient conditions and their fabrication generates minimum waste, where the result is biodegradable and is recycled by nature. Learning how to systematically mimic nature's materials and fabrication processes will dramatically expand our capabilities and choices as well as improve our ability to recycle materials and protect the environment. Also, it can benefit humans in many other ways including the development of more life-like prosthetics as well as artificial hips, teeth, structural support of bones and others.

Biological structures are made either as an integral part of the body, including the bones, shells, and nails; or made by them to support the life essentials including bird nests, cocoon shells, spider web, and underground tunnels. These structures have numerous advantages such as resilience, multi-functionality, and great fracture toughness. The spider, bees and beaver are examples of creatures that produce amazing structures and demonstrate great engineering skill. The spider creates large flat webs; the beavers construct large habitats on water streams and the bees produce the honeycomb that is a highly efficient packing configuration for laying their eggs with nurturing material (the honey) for their off-springs [13]. The honeycomb structure is also made by humans and it is widely used to create aircraft structures benefiting from the low weight and high strength.

\subsection{Senses and Sensors}

An important aspect of active systems is the ability to control the response in order to maintain the critical functions and assure effective performance. To do so such systems need sensing capability and the senses/receptors of biological systems are an important model for mimicking and inspiration. The senses are providing inputs to the central nervous system about the environment around and within their body and the muscles are commanded to act based on the analysis of the received information [14]. Biological sensory systems are extremely sensitive and limited only by quantum effects [5, 6, 15, and 16]. Similarly, sensors are critical to the ability of systems' to monitor their functions and allowing timely response to changing conditions [16]. They are widely used and no system can be imagined to operate effectively without them. Pressure, temperature, optical and acoustical sensors are widely used and continuously being improved while reducing their size and consumed power.

As our body monitors the temperature and keeps it within healthy limits, our homes, offices, and other enclosed living areas have environmental control that operates them at comfortable temperature range. Moreover, the senses of smell and taste are increasingly being mimicked using artificialnose and artificial-tongue, respectively. Understanding the sense of smell led in 2004 the two researchers, Linda B. Buck and Richard Axel [17], to receive the Nobel Prize Award. The sense of smell makes chemical analysis of airborne molecules to determine presence of danger, hazardous chemicals, as well as enjoy good food and other pleasant odors. Developments of artificial noses were reported since the mid-1980s [18], and some of the applications of the current commercial devices include monitor the environment in various facilities and quality control of food processing. The sense of taste is also a biological analyzer of chemicals - it identifies dissolved molecules and ions [19], and similar to the artificial nose, researchers are developing artificial tongues [20]. Artificial tongues, also known as electronic tongue or E-tongues, are used to monitor environmental 
pollution, search for chemical/biological weapon, drugs, and explosives, monitor food taste and quality, and perform non-invasive medical diagnostics (including tasting patient's breath, as well as analyzing urine, sweat, and skin odor). Other mimicked senses include the vision in the form of cameras (Figure 4), where air is used instead of the fovea aqueous content; the whiskers of rodents are mimicked as collision avoidance sensors; and acoustic detectors imitate the sonar in bats and dolphins.

Humanlike robots are equipped with many of biomimetic sensors. For example, audio and visual sensors are used to sense the robot's environment and help in its interaction with humans and negotiating mobility next to adjacent objects. Video cameras are used to determine the robots location and surrounding content as well as providing communication cues from human facial expressions to help the robot act sociably. Using acoustic sensors, robots sense sound to determine the content and direction of received acoustic waves as well as support speech recognition for natural verbal communication. In terms of bandwidth, sensitivity and resolution, the biological hearing sense is far superior to any human-made sensors. It is interesting to note that, for protection against potential danger, hearing is the most important sense since missing an indication of an approaching predator can make it too late by the time other senses receive indication. Advances in understanding hearing led to the development of effective cochlear implants providing deaf people an electrical stimulation directly to the auditory nerve, bypassing the damaged cochlea that causes the deafness [21].

\subsection{Artificial muscles and actuators}

Biological muscles were optimized over millions of years of evolution and, therefore, effectively they are the same mechanism in all the biological systems except for bacteria. Muscles are driven by a complex linear mechanism that allows them to lift large loads with short time response in the range of milliseconds [22]. Functionally, actuators emulate biological muscles but they have many limitations compared to muscles where hydraulic actuators are heavy and have low efficiency; electric motors have limited power density; and combustionenginesarebulkyandneedcontinuousoperation. Active materials are widely used as actuators [23] and they include shape memory alloys [24], electroactive polymers [6, 25], ferroelectric materials [26], and magnetostrictive compounds [27]. Their use involves significant design challenges where, for example, piezoelectric actuators [28] require mechanical amplification in order to increase their very small displacements and benefit from their high power densities.

Electroactive polymer (EAP) actuators are the closest to mimic natural muscles and for this capability they received the moniker "artificial muscles" $[6,25]$. The generated displacement can be designed geometrically to act as actuators that bend, stretch or contract. Today, there are many known types of EAP materials and most of them have emerged in the 1990s. The author divided the various EAP materials into two groups distinguishing them by their operation mechanism:

- Ionic EAP: These polymers consist of two electrodes and electrolyte. Electrical activation causes transport or diffusion of ions resulting in bending or elongation of certain materials and configurations of this group [29]. Examples of these materials include ionic polymermetal composites (IPMC), conducting polymers, carbon nanotubes, and ionic polymer gels. The ionic EAP materials generate large or moderate to large bending strain under low activation voltage (1-2 Volts). However, they exhibit slow response due to the dependence on the diffusion of charges, they need to maintain electrolytes wetness, they have relatively low efficiency in the range of $\sim 1 \%$, and the gels and IPMCs materials have difficulties sustaining constant displacement under activation of a DC voltage.

- Field-activated EAP: This EAP group is activated via the Coulomb force generated by an electric field between the electrodes of a polymer film and examples include
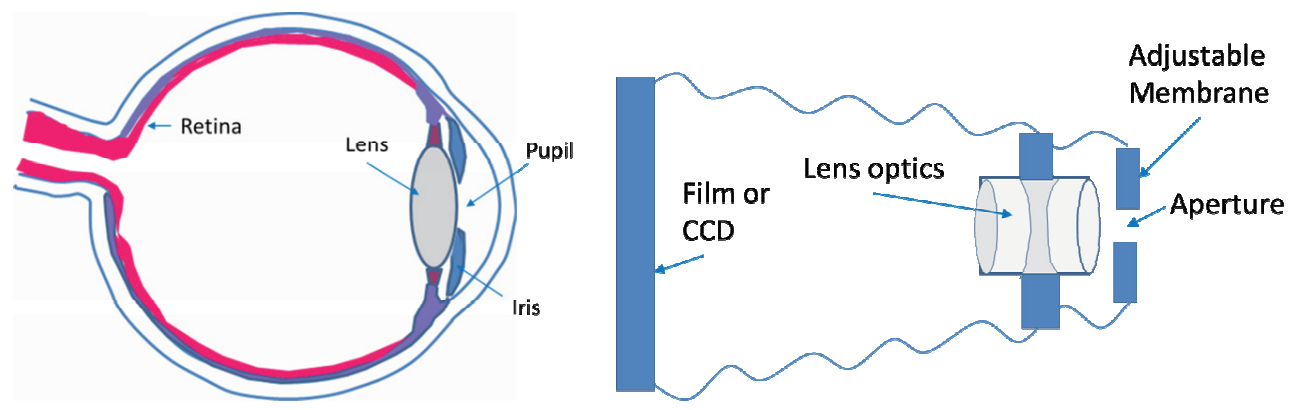

Fig. 4. The human eye (left) and a sketch of a camera (right) as a mimicked optical sensor 
electrostrictive, piezoelectric, and ferroelectric. Since the actuation does not involve diffusion of charge species these polymers can respond as fast as milliseconds. Under a DC voltage they can hold induced displacement, they have a greater mechanical energy density than the ionic EAP group and they can be activated in air with no major constraints. However, they require high activation field that may be close to the electric breakdown level.

Potentially, EAP actuators offer the capability to produce biomimetic "soft" robots however the forces that they can generate in practical actuators are still far from what can be achieved by human muscles [25]. To help accelerate advances in this field, the author initiated and organized in March 1999 the first annual international EAP Actuators and Devices (EAPAD) Conference [30], which is part of the SPIE's Smart Structures and Materials Symposium. At the opening of the 1st Conference, the author posed a challenge to the worldwide scientists and engineers to develop a robotic arm that is actuated by artificial muscles to win an armwrestling match against a human opponent (see its icon in Figure 5). On March 7, 2005, the author organized the first arm-wrestling match with human (17-year old high school female student) as part of the EAP-in-Action Session of this Conference (Figure 6). The student easily won against all the participating three arms and it demonstrated the weakness of the current materials. In a future conference, if advances in EAP lead to sufficiently high force, a professional wrestler will be invited for another human/machine wrestling match.

\section{Biomimetic mechanisms}

Nature is filled with biological mechanisms and the

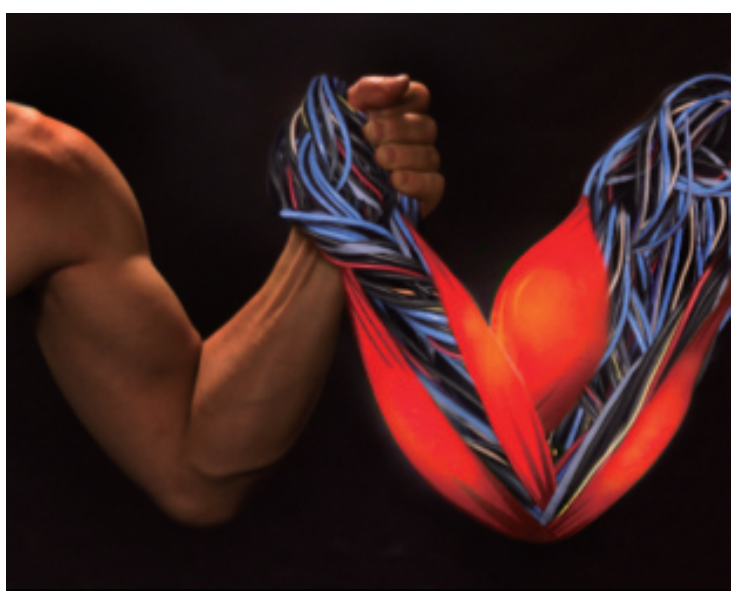

Fig. 5. The icon of the grand challenge for the development of EAP actuators. following are some of the examples of ones that were mimicked.

\subsection{Pumping mechanisms}

Pumping mechanisms [31] are widely used in nature and they inspired many human-made designs. The most common method is the peristaltic pumping where liquid is squeezed in the required direction. Another type is the heart of humans and animals where valves and volume changing chambers and used - the chambers expand and contract and thus controlling the blood flow thru the veins and arteries. Breathing via our lungs is made by pumping air in a tidal process using diaphragm movement generated by the intercostals muscles. Using capillary forces, plants pump water and minerals and distribute them evenly independent of the height, which can reach many meters. This pumping system in plants is capable of delivering very large forces allowing tree roots to fracture rocks. While nature's pumping mechanisms have been mimicked in various devices the high efficiency of the biological pumps offer great models for improved mimicking.

\subsection{Defense and attack mechanisms and devices}

Besides camouflage, predators use many other techniques to catch preys while the latter use various techniques to avoid being haunted. Some of the capabilities have already been duplicated in human-made tools while others are still a challenge to mimic. The suction caps, which are part of the octopus tentacles, are used to strongly grip on preys and they were widely mimicked for such applications as mounting objects on the smooth surfaces of tiles and glass windows. On the other hand, it is currently impossible to mimic the

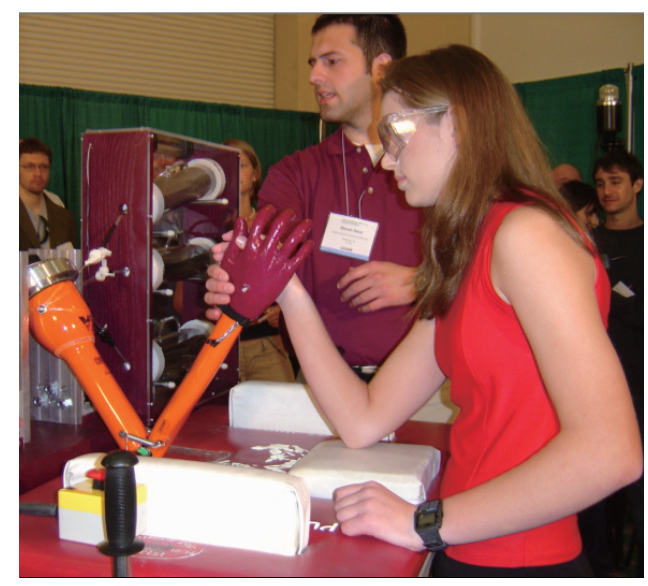

Fig. 6. An EAP driven arm made by students from Virginia Tech and the human opponent, 17-year old student. 
octopus incredible capability to match the color, pattern, shape and texture of the adjacent objects as well as the ability to traverse through narrow openings that are far smaller than its body's normal cross section. For future biomimetic applications, one can imagine the development of a "spy robot" that can be used to enter a room thru the gap under the door by reconfiguring the body shape and then match the colors and texture of the floor covering and maintain low profile to minimize visibility. Such an imaginary robot could perform tasks of finding and catching wanted criminals and terrorists by employing tentacles to hold suspects.

Other potential biological inspiration is the ability of ants to identify food from great distance - a biomimetic approach can be to make swarms of ant-like micro-robots equipped with artificial nose that could be developed to search for residues of explosives and illegal substances. Such robots may perform continuous surveillance, notify the authorities and possibly perform critical explosive disabling functions.

Camouflage is not only the ability to make the body "invisible" but also a method of creating an illusion about the body dimensions to increase the deterrence. Examples include insects or animals making their body appear larger than their actual size and, throughout history, armies have used this tactic to win wars against their enemies.

\section{Biomimetic mobility in water and air}

Propulsion and mobility mechanisms can be inspired by biomimetic characteristics for operating in hard to reach areas [6]. Generally, animals are able to operate almost anywhere on Earth and to negotiate with fluidic performance highly varied environments. An example of a biomimetic robot that was designed to operate in hard to reach areas is the RoboLobster [32], which was developed for searching mines buried beneath beaches or floating in shallow waters. The conditions at the locations where mines are expected to be hidden are considered too harsh for the general types of marine creatures. On the other hand, biological lobsters are able to operate in such environments and mimicking them can potentially provide useful mine detection capabilities.

\subsection{Mobility in water}

Swimming is a biological propulsion method of traversing through liquid media and it is used by all the marine species and many organisms [6]. The jellyfish uses passive swimming employing water currents to float and do not exert energy to control the position or motion. In contrast, active swimming consists of body movement to travel in the desired direction and the speed can reach over $75 \mathrm{~km} /$ hour (e.g., billfish and sailfish). Biological swimmers are inspiring designs of many water mobility capabilities and the fins are an example. They are widely used by swimmer and divers to improve the performance. The stability and maneuvering of swimming species in underwater conditions is determined by the morphology, position and mobility of their control surfaces. Increased understanding and the development of related analytical tools is enabling improvements of the design of watercrafts and other marine vehicles as well as the application of hydrodynamic capabilities that are biomimetic [33].

\subsection{Biologically inspired flight}

The enormously large number of flying capable species suggests that nature has been quite successful with its aerodynamics related "experiments". Flight of insects and birds has been a source of inspiration to human and for many years it was a great challenge [34]. Birds are able to maneuver in flight with amazing performance and they can carry relatively large and heavy food/prey as well as fly to great distances in their seasonal migration. They can even catch preys while flying and an example is the hawk ability to catch a running rabbit. Moreover, birds are able to catch fish by diving into water while correcting for the light path thru the water surface. Mastering flight became possible only after the principles of aerodynamics were better understood. Today, there is no flying creature that can fly as far, carry as much weight with so large body and operate in so extreme environments as the aircraft and other flying machines that are currently being made (Figure 7). However, in spite of the success of today's aerospace technology that ranges from huge transport aircraft with intercontinental destinations to micro-air vehicles, it is still a great challenge to mimic the flying performance of the dragonfly and the hummingbird (Figure 8), or the ability to mimic the Monarch butterfly with a micro-air vehicle that can traverse enormous distances using relatively low power.

Efforts to create other flying mechanisms are ongoing

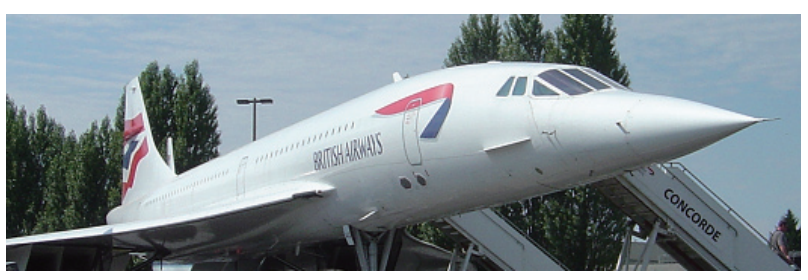

Fig. 7. Using aerodynamic principles led to the development of such aircraft as the supersonic passenger plane, the Concord. Photographed by the author at the Boeing Aerospace Museum, Seattle, WA. 
by many inventors and examples of successes include the gliders and the wingsuits. The latter is a special jumpsuit that turns the human body into an airfoil that creates lift [35]. It is made of fabric that uses the legs and arms as structural elements of the airfoil and allows a person to mimic the flying of such animals as the flying squirrel. The flying person manipulates the body to control the lift and drag by changing the shape of the torso, arching or bending at the shoulders, hips, and knees, the tension on the fabric wings of the suit, as well as the angle of attack in which the wingsuit flies relative to the wind. These maneuvers allow for reduced vertical speed to prolong the free-fall and maximize the horizontal glided distance. The user starts the flight by jumping from a sufficiently high altitude and to end the flight a parachute is opened to significantly slow the landing speed. In contrast, the flying squirrel uses its tail as a rudder for slowing its flight speed.

Producing birdlikeflyingmechanisms is beinginvestigated by various scientists and engineers [34]. At the Ohio Aerospace Institute a study is taking place to enable flying at very low Reynolds number regime on Mars and other planetary missions. An entomopter vehicle was proposed to achieve substantially higher lift by designing a biomimetic configuration and use circulation control techniques (Figure 9). The concept is based on the use of a micro-scale vortex at the wing's leading edge as determined in 1994 by Charles Ellington of the University of Cambridge [36]. Researchers at the Georgia Tech Research Institute have made a preliminary confirmation that this concept may be feasible for taking off, flying slowly, hovering, and landing on Mars.

Birds are able to adjust their wings shape to control the flying speed, gain great maneuverability and reduce the required energy has been sought by various investigators. To mimic these capabilities in the form of an aircraft with morphing wings has been a project that was funded by NASA Langley Research Center (LaRC) [37]. The goal has been to develop such wings design in order to reduce fuel consumption, enhance the maneuverability and enable faster flights over longer distances. Results have shown that

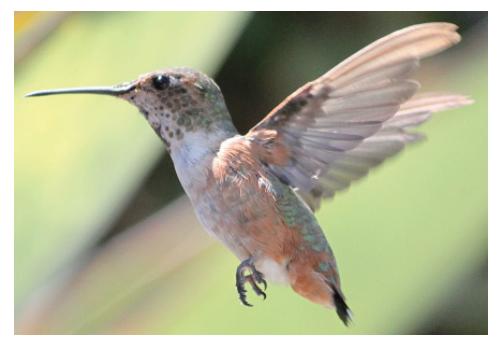

Fig. 8. The hummingbird is a very capable flyer. It performs precision tasks of inserting its beak into flowers and sucking nectar while hovering. in-flight airfoil shape modification allows for drag reduction and delay the flow transition from laminar to turbulent by moving the transition point close to the wing trailing edge [38].

Alternative to flapping wings as a form of propulsion in air, wagging the body and tail has also been considered. Using helium filled balloon design (Figure 10), researchers at EMPA, Switzerland, Duebendorf, Switzerland, in collaboration with the Institute of Mechanical Systems of ETH, Zürich, Switzerland, developed a fish-like propelled blimp [3940]. The actuation is done by electroactive polymers (EAP) emulating muscles, where initially a blimp was developed with flapping fins. A more recent progress of this research has been the development and demonstration of a blimp with wagging body and tail $[6,41]$.

\section{Robotics as beneficiary of biomimetic technologies}

Robots are electromechanical devices that have biomimetic characteristics and they have great advantages

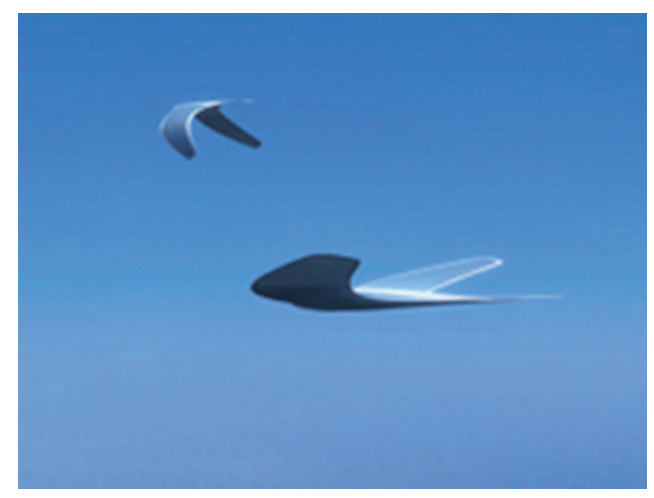

Fig. 9. A flying mechanism that mimics a bird was proposed for planetary exploration missions. Courtesy of Anthony Colozza, Ohio Aerospace Institute.

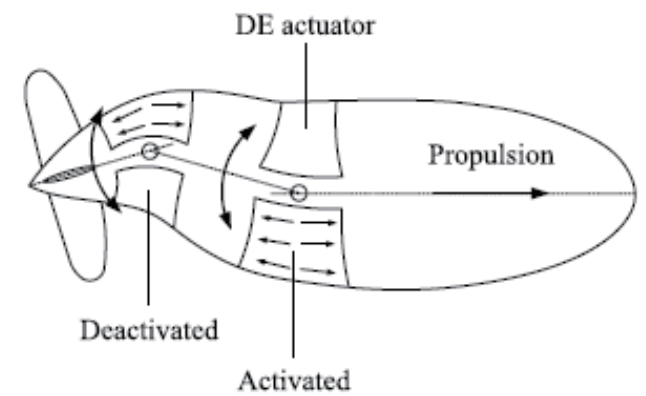

Fig. 10. A graphic view of the EAP activated blimp propelled by wagging the body and tail just like a fish. Courtesy of Silvain Michel, EMPA, Materials Science \& Technology, Duebendorf, Switzerland. 
in performing complex tasks, operating in hard to reach areas and in conditions that are too harsh or dangerous for humans [6]. While wheeled vehicles are quite capable of moving at high speed and low power when the terrain is a paved road, they are highly constrained in such natural areas as steep cliffs and dunes. Robots that traverse terrains via the use of legs are increasingly being developed and they are even considered for space and military applications (see examples in Figure 11 and Figure 12). For planetary exploration of steep terrains, a 4 legs rover was developed [42] using ultrasonic/sonic anchors [43, 44]. This anchoring mechanism allows the rover to "hang-on" rocks by drilling into them via relatively low axial force and, when ready to move, the anchor is extracted by applying a reverse action [45]. Alternative methods of traversing on walls have been studied using the gecko lizards as a model [3]. The millions of tiny, flexible hairs on the gecko's feet apply van der Waals attraction forces that provide powerful adhesion to walls that are as smooth as glass. To mimic this capability tapes with nanoscopic hairs are being developed for various applications.

The ability of robotic vehicles to traverse complex terrain is limited by the lack of intelligent control that coordinates the required ground reaction forces. Developing the required control systems will enable robotic vehicles, with legged ones in particular, to operate in natural terrain with severe obstacles and unanticipated perturbations. The mobility of highly adaptive mammals, reptiles, and insects, offers a mimicking model for developing high levels sensory feedback and mechanisms to enable such mobility. It may be able to climb trees like monkey, run very fast like a cheetah, and hop to great heights and distances like the kangaroo or the grass hopper. Such vehicles will need to be lightweight and consume as little power as possible.

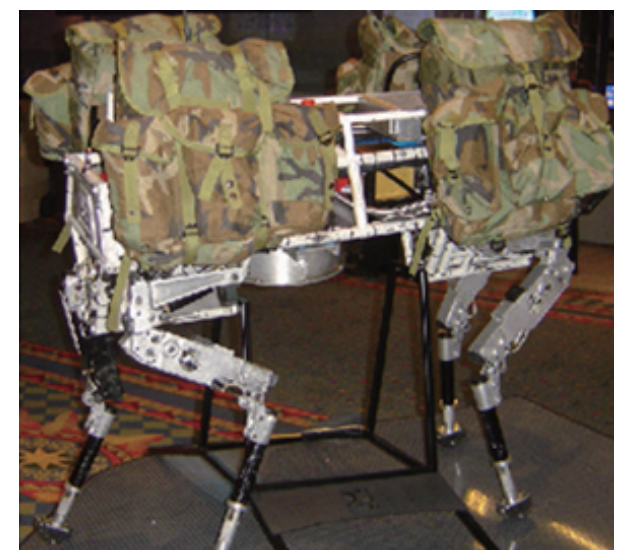

Fig. 11. The Big-Dog (made by Boston Dynamics), which is a mulllike legged robot that was developed for military application. Photographed by the author.
Industry is increasingly benefiting from the advances in robotics and related biologically inspired automation $[7,46]$. Crawlers with manipulation devices are used to perform a variety of nondestructive evaluation (NDE) tasks. At JPL, a multifunctional automated crawling system (MACS) was developed for rapid scanning of aircraft structures in field conditions. MACS consists of two legs for the mobility on structures with one of the legs designed also to rotate. This crawler performs scanning by "walking" on aircraft fuselages while adhering to the surface using suction cups and is capable of walking upside down on various structures. The mobility on structures critically dependents on having controlled adherence and various alternative methods are being used including magnetic wheels [47].

\subsection{Humanlike Robots}

Mimicking humans is an incredibly complex challenge. Advances in materials, artificial intelligence, actuators, communication, speech synthesis, image and speech recognition, and many other capabilities are making it increasingly more feasible to create lifelike robots $[6,7$, and 46]. Depending on their degree of similarity to humans, the author has used the following terms to describe such robots:

Humanoids are robots that mimic the general appearance of humans and they include a head, arms, and possibly legs and eyes. Generally, the head may be shaped as a helmet, and these robots are easier to make than exactly copying the external human form. Humanoids are already quite developed and some are available commercially.

Humanlike Robots are designed to appear as close to real humans as possible and great efforts are made to exactly copy the human appearance and performance. Such robots

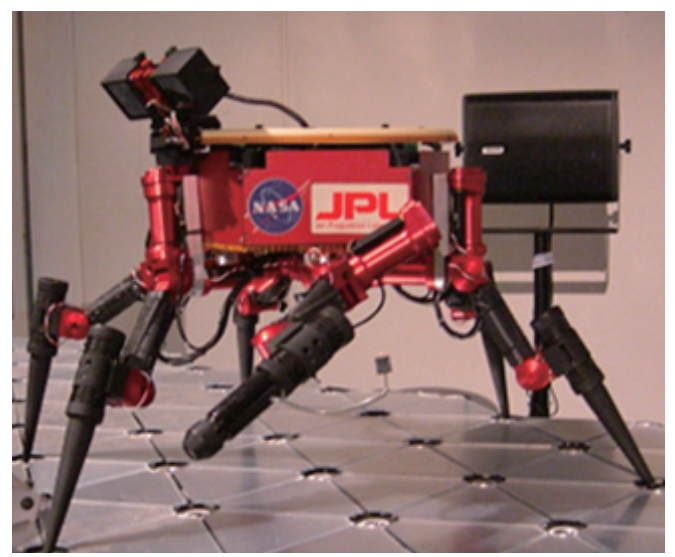

Fig. 12. A 6-legged robot developed at JPL for potential application in future NASA exploration missions. 
are made mostly by roboticists in Japan, Korea and China, with a few in the USA. An example of a humanlike robot is shown in Figure 13, where the similarity of the roboticist Zou Renti, China and his clone robot are quite impressive.

As the capabilities of humanlike robots improve they are expected to enter our life in the form of either household appliances, or perhaps even human peers; they may replace unskilled human labor, or possibly perform difficult and complex tasks in hazardous conditions. However, such humanlike machines may raise concerns, fear and dislike [46]. The Japanese roboticist Masahiro Mori [48] hypothesized that as the degree of similarity between robots and humans increases there will be initial enthusiasm, but as the similarity becomes closer it will turn into strong rejection and dislike, and finally as the likeness becomes very close there will be favorable attitude shift. Mori [48] described these attitude shifts graphically with a dip on a continuous curve, which has become known as the Uncanny Valley hypothesis. The fear of humanlike robots may also be related to our natural sensitivity to minute behavioral anomalies that may possibly indicate an illness. This may be part of our nature as living creatures and the survival of the fittest making us sensitive to genetic disorders, which raises an unconscious alarm of the potential impact on the gene pool. Critics do not accept this hypothesis as fact and they argue that it has never been proven by systematic experiment.

Sociable humanlike robots are increasingly being developed with impressive capabilities including verbally and facially express emotions as well as respond emotionally [46]. Making humanlike robots with social skills can have many important benefits including insights into human behavior providing psychologists with effective tools for treating many phobias and deficiencies in communication skill. Recent advances in virtual reality and AI are having an

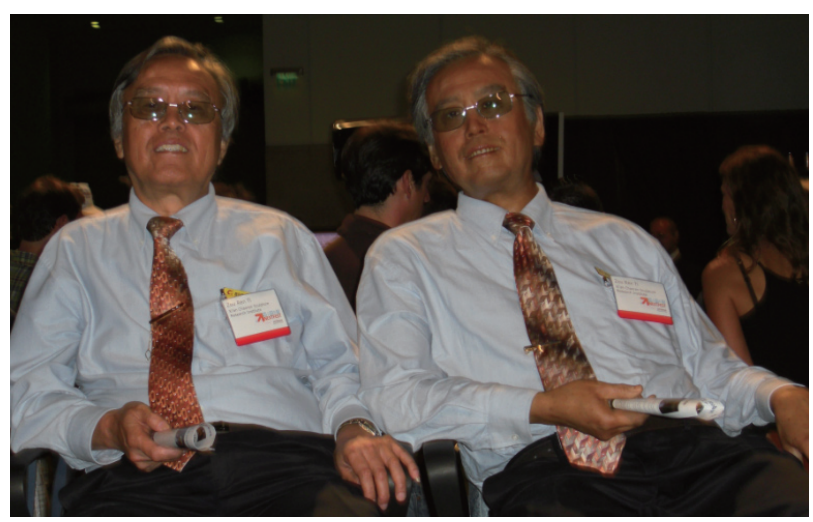

Fig. 13. An example of a humanlike robot showing the roboticist Zou Renti, China, and his clone robot. Photographed at the Wired Magazine 2007 NextFest that was held in Los Angeles, CA, on Sept. 14, 2007. impact on methods of treating children with autism as well as patients with such phobias as fear of height, and closed areas.

\subsection{Micro-robots}

Micro-robotics is increasingly being developed as a result of advances in Micro-Electro-Mechanical System (MEMS). The development of tiny self-propelled micro-robots that can travel through the bloodstream and conduct micro-surgeries was initiated in the 1970s by the Defense Advanced Research Projects Agency (DARPA). Various ideas were explored for medical diagnostics and some have emerged in commercial devices including the pill-like capsules, the PillCam, which are made by the Israeli company, Given Imaging. This capsule is swallowed by the patient and it records images as it travels thru the gastrointestinal (GI) tract and propelled by the body's own natural peristaltic forces. Along the way, it takes videos from the walls of such organs as the small intestine and the esophagus. While the current capsules have no propulsion capability there are various on-going studies that are seeking to develop mobile types [e.g., Refs. 5 and 49]. The development of the "Artificial Bacterial Flagella" $(\mathrm{ABF})$ at ETH, Zurich, Switzerland, brings the original DARPA initiative closer to realization. This micro-robot is made by MEMS fabrication methods and it is capable of controlled swimming closely resembling flagella bacteria [50]. The ABF length is $25-65 \mu \mathrm{m}$ and it is using a spiral tail for propulsion. It is steered to specific targets by tuning the strength and direction of a rotating magnetic field generated by an electromagnet. The ABF is capable of moving forward and backward, upward and downward, as well as rotate. While significant advances were made, we are still at the early stages of developing the capability for a micro-robot to be functional and controllable enough to safely perform operations inside a human body.

\subsection{Novel toys}

The entertainment and toy industries have greatly benefited from advances in robotics. Toys that mimic the appearance and movement of such creatures as frogs, fish, dogs and even babies are now part of many stores. Also, higher-end humanlike robots toys are becoming increasingly sophisticated, and they are designed to walk and even converse with humans using a vocabulary at the level of hundreds of words [6]. Some of the robots can be operated autonomously or remotely reprogrammed to change their characteristic behavior. Generally, toys are a great testbed for new technologies since they are not expected to have the durability that other products are required to meet. The 
turning of an idea to an application can be quite fast and successful toys can bring sufficient revenues to support further development of many other toys that are designed with new technologies. Many biomimetic ideas have been applied to toys including the Transformers that have attracted the attention of many boys. An example is the Bakugan that in its packed forms has the shape of a small ball about the size of a gulf ball and when opened it has various selected shapes. It is interesting to note the similarity of the Bakugan concept and the insect Armadillidium vulgare, also known as the "roly poly". The latter has a shape of a ball when it activates its defense mechanism and it is a multi-legged insect when it feels sufficiently safe.

There are many examples of nature's inventions that could be adapted to produce novel and exciting toys. However, the most difficult mimicking challenge is the four forms that butterflies take during their life cycle: egg, caterpillar, cocoon and the butterfly. Making an artificial reconfigurable mechanism that matches this capability is far beyond what is feasible today or the foreseeable future.

\section{Medical Applications}

There are applications of biomimetics to numerous fields and the medical field was chosen here as an example. It has been greatly inspired by nature and many capabilities were developed by bio-mimicking but there are many possibilities that are still far beyond our technologies capability. Regrowing maimed organs can benefit humans enormously and it exists in such species as the lizard, which sheds its tail as part of its defense mechanism of decoy and it regrows it soon afterward. Efforts to enable such a re-growth are underway including the use of extracellular matrix materials [51] that sometimes triggers the regeneration that humans are capable of. Also, the use of stem-cells is being investigated for such regeneration capabilities.

Certain animals evolved to survive quite extreme conditions including the bears' hibernation for as long as 7 months surviving the extreme cold and lack of food in the winter [52]. Another type of survival mechanism is known as the estivation or aestivation, which is a state of dormancy that occurs during times of extremely hot and dryness conditions that may include drought, and it allows species such as the desert snails to survive up to 5 years without water. Although these survival mechanisms need further studies, they can inspire important medical capabilities. The ability of species to slow their body's metabolism to almost zero offers an important capability that if mimicked may allow for military casualties and accident victims to survive until adequate medical assistance is available. Patients with terminal diseases may be kept alive till potential cure is developed. Also, people that suffer kidney problems that require dialysis may benefit from the capability of the bear to hold the urine in the body for extended periods without damage to the body or health deterioration.

\section{Biomimetics - potential revolution in tech- nology}

Evolution over millions of years led to inventions that are great models for mimicking and inspiration of new technologies. These inventions involve all the sciences and engineering disciplines, and a wide range of scales from as small as nanometers. Mimicking such inventions offers many potential benefits to our life and it is becoming easier as better tools are emerging. The common design requirements of engineering structures are quite similar to those of biological systems including the need to have a structure that is light weight, consume minimum power, and is durable over the life of the species or product [53]. In the case of biology, a failed design leads to extinction of the specific species while in the case of engineering structures they are retired from service and the design is modified or eliminated from use. Biomimetics may consist of simply copying and an example is the spider web that most likely led to the fabrication of the fishing nets. Others may provide inspiration such as the observation of insects and birds flight suggesting that it is possible to fly but achieving it necessitated the development of the related critical knowledge and knowhow. Scientific approaches are helping humans understand nature's capabilities and principles leading to the development of highly effective mechanisms, tools, algorithms, and approaches.

As opposed to the efforts of engineers to produce identical products in mass production and great precision nature produces duplicates of the same species with quite a bit of differences. It is interesting to note that in spite of the differences and even imperfections the individual members of the specific species perform very similarly.

It is hard to image an effective operation of systems without sensors and biological senses and receptors have great capabilities for mimicking. It may be productive to examine the many intriguing sensing capabilities developed by evolution and seek to both mimic the capability and consider related applications. Examples of such senses are animals' ability to sense upcoming nature's disasters, such as earthquake and tsunami. Various observations suggest that certain fish, rodents, snakes and even toads are able to 
sense coming earthquake [53]. Moreover, it is interesting to note that when a big tsunami hit the pacific region in 2004 many animals fled to high ground while humans did not have a clue about the meaning of the receding seawater and the danger that came next.

Nature provides an important guide to living in harmony with it [3]. For example, we can learn from plants how to use pollution on Earth in the form of $\mathrm{CO} 2$ to produce oxygen, thus pollution is converted to a critical resource. Also, plants harvest solar energy in an Earth friendly form and green forms of energy harvesting are increasingly being developed and commercialized and many ideas could be adapted by mimicking or being inspired by nature.

Success in developing and implementing nature's ideas can transform science fiction and imagination into engineering reality. However, there are many challenges to biomimetics including the making of octopus-like robots. This would require high dexterity, intelligence and autonomy with the capability to traverse through very narrow openings; camouflage the body to match the colors, shape and texture of the surrounding; having multiple tentacles and suction cups for gripping on objects; using ink to form smoke screen; and perform simultaneously multiple tasks. The level of complexity that these challenges are involved can be further appreciated with the current impossibility of making robots that grow from a micron-size "seed" in a dormant state (as the seeds of plants) to a fully grown, active machine that operates autonomously. It is hard to predict what would be learned or mimicked next but one can envision in the years to come many more tools and capabilities will emerge at every scale of our life. Areas such as medical, military, consumer products and many others may potentially see considerable benefits from revolutionary technology that biomimetics will introduce.

Implementing innovation based on nature can result in such benefits as improved drugs, stronger and multifunctional materials, and superior robots. This suggests that it is important to preserve Nature's solutions and we need to assure that species are not extinct since they may harbor inventions that we have not been well understood yet. We can learn manufacturing techniques from animals and plants including the use of sunlight and materials production with no pollution, including the development of biodegradable fibers, ceramics, plastics, and various chemicals. One may develop extremely strong fibers that are like the spider web, and ceramics that are shatterproof like the pearl or seashells. Nature also provides a guide as far as the appropriateness of our innovations in terms of durability, performance, and compatibility.

\section{Acknowledgements}

Some of the research reported in this paper was conducted at the Jet Propulsion Laboratory (JPL), California Institute of Technology, under a contract with National Aeronautics and Space Administration (NASA).

\section{References}

[1] Mann S., Biomimetic Materials Chemistry, Wiley Publishers, Hoboken, NJ, 1995, pp. 1-400.

[2] Nemat-Nasser S., Plaisted T., Starr A., and VakilAmirkhizi A., "Multifunctional materials", BiomimeticsBiologically Inspired Technologies, edited by Bar-Cohen, Y., CRC Press, Boca Raton, FL, 2005, pp. 309-341.

[3] Benyus J. M., Biomimicry: Innovation Inspired by Nature, Perennial (HarperCollins) Press, New York, NY, 1998, pp. 1-302.

[4] Vincent, J. F. V., “Stealing Ideas from Nature," Deployable structures, edited by Pellegrino S., Springer-Verlag, Vienna, 2001, pp. 51-58

[5] Bar-Cohen, Y., Biomimetics - Biologically Inspired Technologies, CRC Press, Boca Raton, FL, 2005, pp. 1-527.

[6] Bar-Cohen, Y., Biomimetics: Nature-Based Innovation, CRC Press, Taylor \& Francis Group, Boca Raton, FL, 2011, pp. 1-788.

[7] Bar-Cohen, Y., and Breazeal, C., Biologically-Inspired Intelligent Robots, SPIE Press, Bellingham, Washington, 2003, pp. 1-393.

[8] Kurzweil, R., The Age of Spiritual Machines: When Computers Exceed Human Intelligence, Penguin Press, New York, NY, 1999.

[9] Luger, G. F., Artificial Intelligence: Structures and Strategies for Complex Problem Solving, Pearson Education Publishers, One Lake Street, Upper Saddle River, NJ, 2001, pp. 1-856.

[10] Russell, S. J., and Norvig, P., Artificial Intelligence: A Modern Approach, Pearson Education, One Lake Street, Upper Saddle River, NJ, 2003, pp. 1- 1132.

[11] Amaral, J.F.M., Amaral, J.L.M., Santini, C., Tanscheiot, R., Vellasco, M. and Pacheco, M., "Towards Evolvable Analog Artificial Neural Networks Controllers", Proceedings of the 2004 NASA/DoD Conference on Evolvable Hardware, Seattle, WA, 2004

[12] Carlson, J., Ghaey, S., Moran, S., Tran, C.A., and Kaplan, D. L., "Biological materials in engineering mechanisms," Biomimetics-Biologically Inspired Technologies, edited by Y. Bar-Cohen, CRC Press, Boca Raton, FL, 2005, pp. 365380. 
[13] Gordon, J.E. “The New Science of Strong Materials, or Why You Don't Fall Through the Floor", 2nd Ed., PelicanPenguin, London, 1976, pp. 1-287.

[14] Hughes, H. C., Sensory Exotica a World Beyond Human Experience, MIT Press, Cambridge, MA, 1999, pp. $1-359$

[15] Bialek, W, "Physical limits to sensation and perception", Annual Review of Biophysics, Biophysics Chemistry, Vol. 16, 1987, pp. 455-478.

[16] Bar-Cohen, Y., "Biological Senses as Inspiring Model for Biomimetic Sensors”, IEEE Sensors Journal, Vol. 1, No. 12, 2011, pp. 3194 - 3201. DOI: 10.1109/JSEN.2011. 2167321

[17] Buck, L. and Axel, R., "A Novel Multigene Family May Encode Odorant Receptors: A Molecular Basis for Odor Recognition", Cell, Vol. 65, No. 1, 1991, pp. 175-187.

[18] Bartlett, P. N., and Gardner, J. W., Electronic Noses: Principles and Applications, Oxford University Press, Oxford, UK, 1999.

[19] Craven, M. A., and Gardner, J. W., "Electronic noses - development and future prospects", Trends in Analytical Chemistry, Vol. 15, No. 9, 1996, pp. 486-493.

[20] Krantz-Ruckler, C., Stenberg, M., Winquist, F., and Lundstrom, I., "Electronic tongues for environmental monitoring based on sensor arrays and pattern recognition: a review", Analytica Chimica Acta, Vol. 426, 2001, pp. 217-226 http://dx.doi.org/10.1016/S0003-2670(00)00873-4

[21] Gantz, B. J., Turner, C., Gfeller, K.E., and Lowder, M. W., "Preservation of Hearing in Cochlear Implant Surgery: Advantages of Combined Electrical and Acoustical Speech Processing", Laryngoscope, Vol. 115, 2005, pp. 796-802.

[22] Full, R. J., and Meijir, K., "Metrics of Natural Muscle Function", Electroactive Polymer (EAP) Actuators as Artificial Muscles - Reality, Potential and Challenges, edited by Y. BarCohen, SPIE Press, Bellingham, WA, 2004, pp. 73-89.

[23] Sherrit, S., Bar-Cohen, Y., and Bao, X., "Ultrasonic Materials, Actuators and Motors (USM)", Automation, Miniature Robotics and Sensors for Nondestructive Evaluation and Testing, edited by Y. Bar-Cohen, TONE Series, ASNT, Columbus, OH, 2000, pp. 215-228.

[24] Brailovski, V., Trochu, F., and Galibois, A., Shape Memory Alloys: Fundamentals, Modeling and Industrial Applications, Minerals, Metals, \& Materials Society, 1999.

[25] Bar-Cohen, Y., Electroactive Polymer (EAP) Actuators as Artificial Muscles - Reality, Potential and Challenges, 2nd Edition, SPIE Press, Bellingham, WA, 2004, pp. 1-765.

[26] Uchino, K., Ferroelectric Devices, CRC Press Taylor \& Francis Group, Boca Raton, FL, 2000.

[27] Engdahl, G., and Mayergoyz, I. D., Magnetostrictive compounds, Academic Press, Salt Lake City, UT, 2000.

[28] Sashida, T. and Kenjo, T., Introduction to Ultrasonic
Motors, Oxford University Press, New York, NY, 1993.

[29] Park, I.-S., Jung, K., Kim, D., Kim, S.-M. and Kim, K. J., "Physical principles of ionic polymer-metal composites as electroactive actuators and sensors", Special Issue dedicated to EAP, Materials Research Society, Vol. 33, No. 3, 2008, pp. 190-195.

[30] Bar-Cohen, Y, Proceedings of the first SPIE's Electroactive Polymer Actuators and Devices (EAPAD) Conf., Smart Structures and Materials Symposium, SPIE Press, Bellingham, WA, Vol. 3669, 1999, pp. 1-414.

[31] Hicks, T. G. and Edwards, T. W., Pump Application Engineering, McGraw-Hill Book Company, New York, NY, 1971.

[32] Ayers, J. and Witting, J., "Biomimetic Approaches to the Control of Underwater Walking Machines", Philosophical Transactions of the Royal Society, Vol. 365, No. 1850, 2007, pp. 273-295.

[33] Fish, F. E., "Biomimetics: Determining engineering opportunities from nature", Proceedings of SPIE Biomimetics and Bioinspiration, edited by Bar-Cohen Y., SPIE Press, Bellingham, WA, 2009. http://dx.doi.org/10.1117/12.824106

[34] Kulfan, B. M., and Colozza, A. J., "Biomimetics and Flying Technology”, Biomimetics: Nature-Based Innovation, edited by Bar-Cohen, Y., CRC Press, Taylor \& Francis Group, Boca Raton, FL, 2011, pp. 525-674.

[35] Abrams, M., "Birdmen, Batmen, and Skyflyers: Wingsuits and the Pioneers Who Flew in Them, Fell in Them, and Perfected Them", Crown Publishing Group, New York, NY, 2006.

[36] Scott, P., “A bug's lift", Scientific American, Vol. 280, No. 4, 1999, pp. 51-54.

[37] Wlezien, R.W., Homer, G.C., McGowan, A.R., Padula, S.L., Scott, M.A., Silcox, R.J. and Simpson, J.O., "The Aircraft Morphing Program", Proceedings of the 39th Structures, Structural Dynamics, and Materials Conference and Exhibit, American Institute of Aeronautics and Astronautics (AIAA), Washington, D.C., 1998.

[38] Courchesne1, S., Popov, A. V., and Botez, R. M., "New aeroelastic studies for a morphing wing", Proceedings of the 48th AIAA Aerospace Sciences Meeting Including the New Horizons Forum and Aerospace Exposition, American Institute of Aeronautics and Astronautics (AIAA), Washington, D.C., 2010.

[39] Michel, S., Dürager, C., Zobel, M. and Fink, E., "Electroactive polymers as a novel actuator technology for lighter-than-air vehicles", Proceedings of the SPIE Electroactive Polymer Actuators and Devices (EAPAD), edited by Bar-Cohen Y., SPIE Press, Bellingham, WA, 2007.

[40] Lochmatter, P., "Development of a Shell-like Electroactive Polymer (EAP) Actuator," Ph.D. dissertation, 
Centre of Structure Technologies, ETH, Zurich, Switzerland, 2007.

[41] Jordi, C., S. Michel, A., Bormann, A., Gebhardt, C., and Kovacs, G. M., "Large planar dielectric elastomer actuators for fish-like propulsion of an airship", Proceedings of the SPIE EAPAD Conference, edited by Bar-Cohen Y., SPIE Press, Bellingham, WA, 2010.

[42] Kennedy, B., Okon, A., Aghazarian, H., Badescu, M., Bao, X., Bar-Cohen, Y., Chang, Z., Dabiri, B., Garrett, M., Magnone, L. and Sherrit, S. "Lemur IIb: A Robotic System for Steep Terrain Access", Proceedings of the 8th International Conference on Climbing and Walking Robots, London, UK, 2005.

[43] Badescu, M., Bao, X., Bar-Cohen, Y., Chang, Z., Dabiri, B. E., Kennedy, B. and Sherrit, S., "Adapting the ultrasonic/ sonic driller/corer for walking/climbing robotic applications", Proceeding of the SPIE Smart Structures Conference, edited by Bar-Cohen Y., SPIE Press, Bellingham, WA, 2005.

[44] Bar-Cohen, Y. and Sherrit, S., "Self-Mountable and Extractable Ultrasonic/Sonic Anchor", U.S. Patent, No. 7,156,189, 2007.

[45] Bar-Cohen, Y. and Zacny, K., Drilling in Extreme Environments - Penetration and Sampling on Earth and Other Planets, Wiley - VCH, Hoboken, NJ, 2009.

[46] Bar-Cohen, Y. and Hanson, D., The Coming Robot Revolution - Expectations and Fears About Emerging Intelligent, Humanlike Machines," Springer, New York, NY, 2009.

[47] Bar-Cohen, Y. and Joffe, B., "Magnetically Attached Multifunction Maintenance Rover (MAGMER)," NTR Docket No. 20229, Item No. 9854, 1997.
[48] Mori, M., “The uncanny valley," Energy, Vol. 7, No. 4, 1970, pp. 33-35. (Translated from Japanese to English by K. F. MacDorman and T. Minato)

[49] Allison, E., Kiraly, Z., Springer, G.S. and Van Dam, J., "Design, development and testing of a remote-controlled, stereoscopic (three-dimensional) imaging, self-propelled wireless capsule endoscope", Gastrointestinal Endoscopy, Vol. 63, Issue 5, 2006, pp. AB104.

DOI:10.1016/j.gie.2006.03.111

[50] Zhang, L., Abbott, J. J., Dong, L., Kratochvil, B. E., Bell, D., and Nelson, B. J., "Artificial bacterial flagella: Fabrication and magnetic control", Applied Physics Letters, Vol. 94, Issue 6,; 2009, http://dx.doi.org/10.1063/1.3079655

DOI:10.1063/1.3079655

[51] Shin, H., Jo, S., and Mikos, A.G., "Biomimetic materials for tissue engineering”, Biomaterials, Vol. 24, No. 24, 2003, pp. 4353-4364.

[52] Depre, C., and Vatner, S., "Mechanisms of Cell Survival in Myocardial Hibernation", Trends in Cardiovascular Medicine, Vol. 15, Issue 3, 2005, pp. 101-110.

[53] Grant, R. A., and Halliday, T., "Predicting the unpredictable; evidence of pre-seismic anticipatory behavior in the common toad", J. of Zoology, Vol. 281, 2010, pp. 263271.

DOI 10.1111/j.1469-7998.2010.00700.x

[54] Yen, J., Weissburg, M., Helms, M. and Goel, A., "Biologically Inspired Design: A tool for interdisciplinary education", Biomimetics: Nature-Based Innovation, edited by Bar-Cohen Y., CRC Press, Taylor \& Francis Group, Boca Raton, FL, 2011, pp. 331-360. 